\title{
A minicomputer controlled inelastic and total intensity light scattering spectrometer for polymer characterization
}

\author{
M. Duval and H. J. Coles $\left(^{*}\right)$ \\ C.N.R.S., Centre de Recherches sur les Macromolécules \\ 6, rue Boussingault, 67083 Strasbourg Cedex, France
}

(Reçu le 19 novembre 1979, révisé le 22 avril 1980, accepté le 28 avril 1980)

\begin{abstract}
Résumé. - On décrit un appareil permettant d'évaluer les propriétés statiques et dynamiques des polymères en solution par mesure de l'intensité diffusée totale et quasi élastique. On donne en détail les tests et calibrages effectués en fonction de l'angle de diffusion $\left(\theta=20 \rightarrow 140^{\circ}\right)$ et de la température. Les mesures dynamiques sont traitées par interfaçage avec un mini-ordinateur PDP 11/34 et l'analyse spectrale par transformée de Fourier rapide a été testée jusqu'à $50 \mathrm{kHz}$ sur des sphères de latex et des solutions de polystyrène dans le benzène. Pour ces derniers échantillons sous forme de pelotes statistiques on a comparé résultats statiques et dynamiques entre eux et on les a reliés aux prédictions théoriques récentes.
\end{abstract}

\begin{abstract}
A self contained apparatus has been described that allows both static and dynamic properties of polymers in solution to be measured by total intensity and quasi-elastic light scattering. Details of the testing and calibration have been given both as a function of scattering angle $\left(\theta=20 \rightarrow 140^{\circ}\right)$ and temperature. The dynamic measurements are carried out on line using a PDP 11/34 minicomputer and spectral analysis using fast Fourier Transformation has been carried out up to $50 \mathrm{kHz}$ for polystyrene latex spheres and polystyrene/benzene solutions. For the latter random coil samples comparison has been made between the static and dynamic results and these have been compared with recent theoretical predictions.
\end{abstract}

1. Introduction. - In recent years light scattering has become one of the most useful methods of characterizing macromolecules in solution or suspension [1]. In such studies the intensity of light scattered is measured as a function of angle and concentration and using the Zimm method [2] the weight average molecular weight $M_{\mathrm{w}}$, radius of gyrations $\left(R_{\mathrm{g}}^{2}\right)^{1 / 2}$ and second virial coefficient $A_{2}$ are determined. In these classical or elastic Rayleigh scattering experiments the average (integrated) light level is measured and no attempt is made to resolve the frequency content of the scattered light. Measurement of this frequency information, which arises from concentration fluctuations of the polymer undergoing Brownian motion, allows the diffusion constant $D$ to be determined, and is known as inelastic or quasielastic light scattering [3, 4].

(*) Permanent address : Department of Physics, Schuster Laboratory, University of Manchester, Manchester M13 9PL, U.K.
In principle therefore it should be possible in one experiment to measure both the static and dynamic properties of a polymer system by elastic and inelastic light scattering respectively. Unfortunately these two techniques set different limits on the design of the apparatus in terms of optical precision and thermal and mechanical stabilities. In fact the two measurements are rarely carried out together [5]. Further many of the dynamic studies rely on a hardware approach that limits their usefulness to measuring correlation functions [6] or power spectra [4] only. It is the purpose of this article to describe a self contained apparatus that measures both static and dynamic properties of macromolecules in solution. This apparatus is based on a PDP 11/34 minicomputer for its signal treatment and has the advantage that all data analysis, least square fitting of curves and results are carried out on line and involve no further peripheral components for such mathematical treatment. The problems encountered in the construction, testing and calibration of the apparatus are given in detail and finally results are presented and compared with 
recent theoretical predictions for a flexible polymer system (i.e. polystyrene/benzene).

2. Light scattering considerations. -2.1 StaTIC. - The design requirements of an apparatus suitable for measuring $M_{\mathrm{w}},\left(R_{\mathrm{g}}^{2}\right)^{1 / 2}$ and $A_{2}$ of polymer solutions are documented in the literature [7]. Primarily the light source must be stable, intense and well collimated. The light scattered $I(\theta)$ by a well defined volume of the sample is measured accurately as a function of angle $\theta$ down to small angles. As the amount of scattered light is small compared to the incident light level $\left(\sim 10^{-6}\right)$ this necessitates the use of sensitive photomultipliers as detectors. Further it is advantageous, as scattering follows a $\lambda^{-4}$ wavelength dependence, to use a blue or green light source. Such wavelengths also fall in the range of greatest spectral sensitivity of most photomultipliers. Assuming such an apparatus is available the molecular parameters previously defined are extracted from the angular and concentration variations of the scattered intensities extrapolated to zero angle and concentration [2]. It has been shown for vertically polarized incident light that $[8]$ :

$$
\frac{K c}{R_{\theta}}=\frac{1}{M_{\mathrm{w}}}\left\{1+\frac{q^{2} \bar{R}_{\mathrm{g}}^{2}}{3}+\cdots\right\}+2 A_{2} c+\cdots,
$$

with

$$
\begin{aligned}
K & =\frac{2 \pi^{2}}{\lambda_{0}^{4} N_{\mathrm{A}}}\left(\frac{\mathrm{d} n}{\mathrm{~d} c}\right)^{2} n_{0}^{2}, \\
R_{\theta}^{-1} & =\frac{I_{\mathrm{b}}^{v}}{I_{\theta}^{v} \alpha} \cdot \frac{n_{\mathrm{b}}^{2}}{n_{0}^{2}} \cdot \frac{1+\rho_{\mathrm{u}}}{R_{\mathrm{b}}}, \\
q & =\frac{4 \pi}{\lambda_{0}} n_{0} \sin \frac{\theta}{2},
\end{aligned}
$$

where $c$ is the polymer concentration, $R_{\theta}$ is the excess Rayleigh ratio of the scattering medium for a scattering angle $\theta$. In practice $R_{\theta}$ is determined, from equation (3), relative to a standard of known Rayleigh $\left(R_{\mathrm{b}}\right)$ and depolarization $\left(\rho_{\mathrm{u}}\right)$ ratios for unpolarized incident light, and the excess vertically polarized light scattered by the solute $I_{\theta}^{v}$ and the standard $I_{\mathrm{b}}^{v}$ at $\theta=\pi / 2, n_{0}$ and $n_{\mathrm{b}}$ are the solution and standard refractive indices, $(\mathrm{d} n / \mathrm{d} c)$ is the solution refractive index increment at the incident wavelength $\lambda$ in the medium $\left(n_{0}\right), \alpha=\sin \theta$ is the volume correction, $N_{\mathrm{A}}$ is the Avogadro number, $q$ is the scattering vector and $\lambda_{0}$ the incident vacuum wavelength.

2.2 Dynamic. - The fluctuations arising from diffusional motion for typical polymer solutions lies in the range $10^{-1} \mathrm{~Hz}$ to $10^{7} \mathrm{~Hz}$. The resolution of such small frequency shifts or line broadening around the scattering line frequency of $10^{15} \mathrm{~Hz}$ has become possible due to the advent of the laser with its high monochromaticity, stability and directionality, and advances in electronic signal processing techniques. Previously such line broadening could only be resolved down to $10^{8} \mathrm{~Hz}$ using optical interferometric techniques. In order to resolve lower frequency shifts two methods have been developed [6, 4]. In the first the digital nature of the photons has been used to count the number of photons scattered at a given angle in a given time interval $(\Delta t)$ as a function of time $t$. From this an autocorrelation function $\langle n(0) . n(t)\rangle$ is determined, where $n(0)$ and $n(t)$ are the number of photons counted at time 0 and $t$ respectively. This autocorrelation function decays exponentially as a function of time and the characteristic decay time $\tau$ is related directly to the translational diffusion constant $\left(D_{t}\right)$. This photon counting technique is particularly useful for measuring low light levels. The second method, used for higher light levels, uses the analog properties of the signal by measuring through Fourier transform techniques the power spectrum $P(v)$ of the temporally fluctuating light level $I(t)$. For a monodisperse polymer system the power spectrum produced is a Lorentzian centred on the laser scattering frequency with a half width at half height $\Gamma$ related directly to the translational diffusion constant $D_{\mathrm{t}}$ by :

$$
\Gamma=D_{\mathrm{t}} q^{2} / \pi
$$

The autocorrelation and Fourier transform methods are complementary, one being the Fourier transform of the other, and thus the dynamic information determined is equivalent.

Most correlation or spectrum analysers are hardware systems that rely on peripheral computers to enable their results to be analysed and thus some of the advantages of the rapidity and relative cheapness are reduced by the need of extra interfaces and post measurement data treatment. As mentioned above the current system uses one PDP 11/34 minicomputer and software routines that allow : (i) the power spectra to be obtained directly (ii) the Lorentzians to be curve fitted and (iii) the straight line depencies of $\Gamma$ on $q^{2}$ and $D_{\mathrm{t}}$ on temperature or concentration (see below), or the extrapolations of the Zimm plot behaviour to zero concentration and angle to be determined.

Recent advances in the calculus of Fourier transforms has led to the development of algorithms allowing the rapid calculation of power spectra using minicomputers. In the present apparatus use of the TukeyCooley algorithm [9] allows the power spectrum $P(v)$ to be calculated through the coefficient $I(v)$ where $P(v)=I(v) I^{*}(v)$ and $I^{*}(v)$ is the complex conjugate of $I(v)$. The function $I(v)$ is the Fourier transformation of $I(t)$, thus

$$
I(v)=\frac{1}{T} \int_{0}^{T} I(t) \mathrm{e}^{-j v t} \mathrm{~d} t
$$

In order to treat the data in the computer the signal $I(t)$ is digitized, using a suitable ADC, into $2 N$ 
channels in time $(2 N=1024)$ and the following expression for $I(v)$ is obtained :

$I(k)=\frac{1}{N} \sum_{i=0}^{N-1} I_{i} \cdot \mathrm{e}^{-j} \frac{2 \pi k_{i}}{N}$ for $k=.0, \ldots, N-1$.

where $N$ is the number of points of the power spectrum, $I_{i}$ is the scattered intensity sampled in the $i$ th channel. Using this format and the Tukey-Cooley algorithm the transform is calculated in $N \log 2 N$ operations rather than the $N^{2}$ operations normally required. It is this that leads to the time saving and is known as the Fast Fourier Transform (FFT). The validity of this method was recently demonstrated [10] in an apparatus that had a frequency limit of $20 \mathrm{kHz}$ and relied on a peripheral computer for data handling. In the current self contained apparatus the sampling frequency range, limited by the ADC is up to $100 \mathrm{kHz}$. Other methods of frequency analysis using band pass filters are possible. Such methods are useful for low frequencies when the number of filters (or data points $N$ ) are low but become extremely expensive for a wide frequency range where the number of filters required will be high. Further the filters must be matched in gain and band width, and the signal output from each of the $N$ filters must be stored for visual display or further data treatment. This would require a supplementary recording/calculating system. The dynamic range of such commercially available frequency analysers is limited to about $20 \mathrm{kHz}$.

3. Experimental. - 3.1 PhySICAL DesCription. A schematic diagram of the quasi-elastic light scattering apparatus is presented in figure 1. Whilst the optical arrangement is fairly classical for such measurements [11] the scattering cell, various electronic components, and the transfer and treatment of the signal are original. Besides the advantages of being self contained and relatively inexpensive compared to equivalent systems the apparatus has the advantages of being very stable mechanically and thermally, performs scattering measurements down to small angles, uses'small sample volumes $(2-3 \mathrm{ml})$, and performs static as well as dynamic measurements. It therefore seems justifiable to dwell in detail on the instrumental factors as well as the problems encountered in building the current apparatus.

3.2 Optical/Mechanical. - 1) A Spectra Physics 165-2 W Argon-ion laser was used as the coherent light source with wavelengths available between 457.9 and $514.5 \mathrm{~nm}$. Normally the $514.5 \mathrm{~nm}$ line in the TEMoo mode was used with a tube current of $\sim 25 \mathrm{~A}$ and in the light stabilized mode. Under these conditions an output of around $700 \mathrm{~mW}$ stable to $0.2 \%$ was obtained. Rather than use the internal aperture to reduce the laser output power we used the external polarizer rotated from the vertical polarization direc-

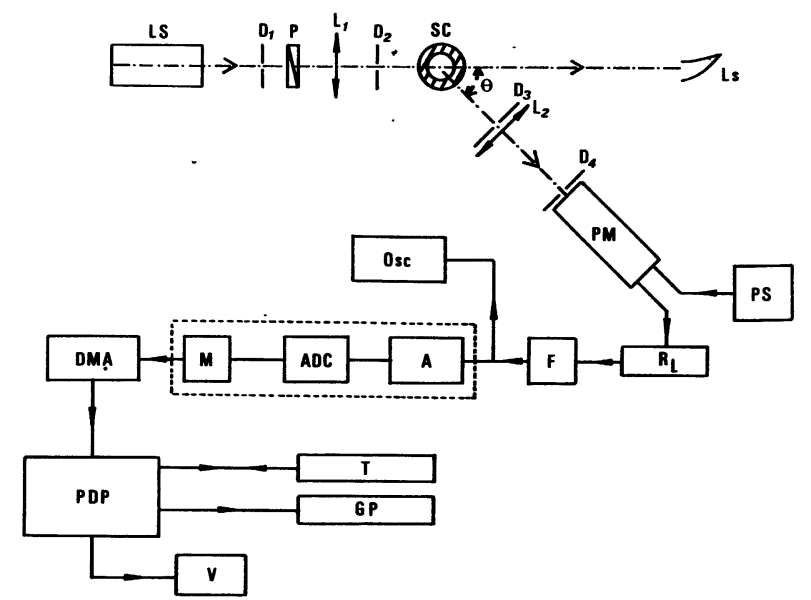

Fig. 1. - Schematic of the light scattering apparatus. LS : laser light source mounted on a positionably adjustable table; $D_{1}$ and $D_{2}$ : variable diameter apertures to minimize back reflections into the laser cavity; $\mathbf{P}$ : Glan-laser polarizer that defines the polarization direction of the incident beam; $L_{1}:$ lens of $20 \mathrm{~cm}$ focal length that focusses the incident beam down to $\sim 100 \mu \mathrm{m}$ in the scattering volume; SC : thermally isolated spectrometer/scattering cell assembly (see Fig. 2) Ls : light stop. The previous components are all mounted on an Oriel type optical rail. The detecting optics are mounted on the rotatable arm of the goniometer platform that supports SC. $D_{3}$ : a pinhole variable between $100 \mu \mathrm{m}$ and $4 \mathrm{~mm}$ that controls the acceptance solid angle and therefore the total light intensity detected by PM the photomultiplier; $L_{2}:$ a lens of $6 \mathrm{~cm}$ focal length that transfers the image of the scattering volume to the entrance aperture $D_{4}$ without magnification (i.e. $\left.\mathrm{SC} \rightarrow L_{2}=L_{2} \rightarrow D_{4}=12 \mathrm{~cm}\right) ; D_{4}:$ an interchangeable aperture that allows the number of coherent areas seen by the photocathode surface to be varied $\left(D_{4}<D_{3}\right.$ and $D_{4} \leqslant$ beam diameter in the scattering cell); PS : high tension photomultiplier power supply; $R_{\mathrm{L}}$ : variable load resistor; Osc : oscilloscope; $\mathrm{F}$ : lowpass filter network; A : amplifier; ADC : analog to digital converter ; $M$ : 8 bit 1024 word memory. A, ADC and M comprise the Datalab recorder. DMA : direct memory access parallel interface ; PDP : minicomputer; $\mathbf{T}$ : command teletype ; GP : graph plotter ; V : minicomputer oscilloscope visualization.

tion of the laser. In fact we found that this aperture and the air spaced etalon when used increased the low frequency noise in the output light signal, although the latter does serve to eliminate mode hopping at higher frequencies [12]. This mode hopping did not occur within our dynamic range of $0-100 \mathrm{kHz}$. Thus we ran our laser without the Fabry-Perot etalon. Two other sources of noise at low frequencies were encountered, $a$ ) that due to the cooling water supply and $b$ ) that at $300 \mathrm{~Hz}$ due to insufficient smoothing in the laser anode current regulating supply. Whilst the noise due to $a$ ) is random and statistically average out that due to $b$ ) is not and further can vary regularly as a function of the gas tube pressure [4]. Fortunately this noise can only be detected when the scattering from the polymer is very weak and if present it is discriminated against during the curve fitting of the data.

2) Scattering photometer. We have designed our own scattering cell holder that allows both low and high angle scattering measurements with small sample 
volumes and a high temperature stability. This is shown in figure 2. The inner tank is filled with xylene that allows refractive index matching with the glass components and thus minimizes boundary refractive effects. Radiating out from the centre of this vat are a series of circular holes that terminate at the flat entrance or exit windows. These holes are drilled at the preselected scattering angle $\theta$ allowing measurements to be made at any angle between $3^{\circ}$ and $15^{\circ}$ and between $20^{\circ}$ to $160^{\circ}$ in $10^{\circ}$ steps. The windows are cut circularly from $0.4 \mathrm{~mm}$ thick optical microscope slides and are supported between teflon washers held firmly in place by circular, hollow grub screws (see inset). With such a system the incident and scattered beams (for $20^{\circ}-160^{\circ}$ ) are always perpendicular to the glass and involve no lensing or birefringent effects. The flat window between $3^{\circ}$ and $15^{\circ}$ is slightly inclined and a small refraction correction has to be made to the measured scattering angle $\theta$. For the straight through beam $\left(\theta=0^{\circ}\right)$ an absorbing black glass light stop is used. The scattering cell with its support which is geometrically centred is essentially that used in the Fica 50 light scattering apparatus. As the height of this cell can be varied and because of the narrow scattering volume of the incident beam the cell can be used with only a few ml's of solution. All inner surfaces of the block were anodized black to eliminate stray reflections. The outer vat contains the temperature regulating fluid, in this case water, which is circulated via a Haake thermostated water reservoir.
With such a system the temperature stability is $<0.01^{\circ} \mathrm{C}$ at up to $30^{\circ} \mathrm{C}$ above the ambient room temperature. The whole scattering cell is insulated by a $1 \mathrm{~cm}$ thick asbestos temperature jacket. The scattering cell was deliberately designed with the maximum amount of metal, and with the cell at the geometric centre, to maximize the thermal stability within the scattering volume. Temperatures were measured using a thermistor that had been previously calibrated against a quartz thermometer. The scattering cell with its holder was then mounted concentrically on the goniometer platform, but with angular adjustment screws that allowed fine rotation of the spectrometer with respect to the goniometer. The goniometer essentially consists of two concentric cylinders, the inner which is static and fixed to the optical rail and the spectrometer and an outer to which is fixed a $30 \mathrm{~cm}$ arm that supports the detecting optics and the photomultiplier on an optical rail. Moving surfaces are separated by roller bearings and the precision of the angular setting is within $\sim 0.1^{\circ}$ for scattering angles greater than $15^{\circ}$. For lower angle scattering a vernier has to be used to increase the an gular precision.

3) The laser, optical components, spectrophotometer and goniometer were all mounted on an Oriel system square cross section optical rail, which was in turn mounted on two adjacent marble slabs $(450 \mathrm{~kg}$ each). In order to eliminate building vibration affecting the apparatus, the whole assembly was supported on

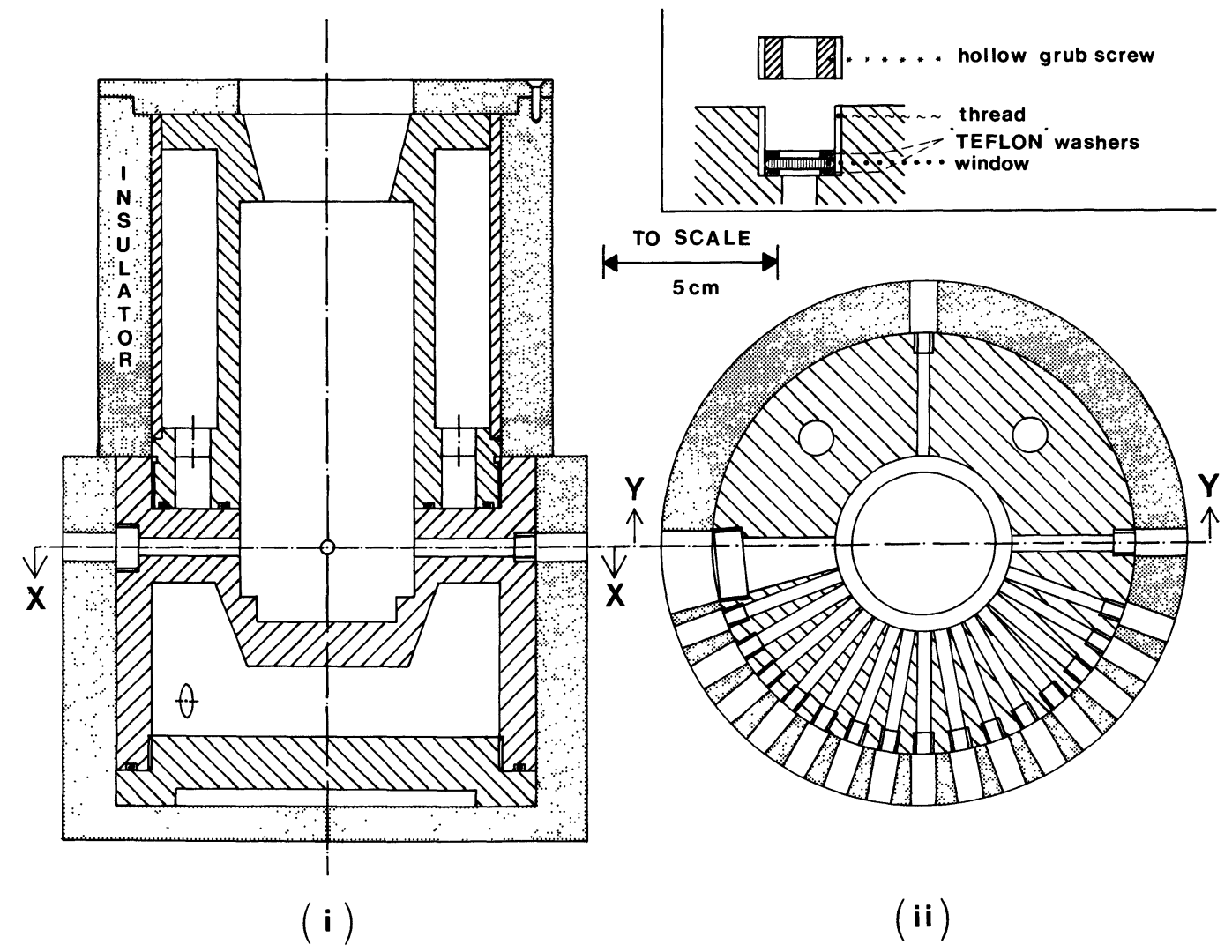

Fig. 2. - The spectrometer cell holder (i) cross sectional elevation through YY; (ii) cross sectional plan through XX. The horizontal centre line marks the laser beam path and the inset shows an enlarged view of the window assembly. 
a cast iron frame isolated from the floor by 6 pillars. These pillars consisted of rubber vibration absorbing pads (Type Radiaflex BNo 521711 absorbing down to $\sim 4 \mathrm{~Hz}$ ) which were in turn mounted on 1 meter high sand columns contained in $(40 \mathrm{~cm}$ diameter $)$ steel drums. This system was free of vibrational effects within the resolution of the apparatus.

3.3 Electronic. - 1) The detecting element is a 12 stage Hamatsu R 585 fast linear photomultiplier with a bialkali photocathode and quartz window giving a spectral response in the range $165.0-650 \mathrm{~nm}$. This PM tube was chosen for its low dark current count rate (5 pulses/s) and its fast pulse rise time $(10 \mathrm{~ns})$. In theory such tubes may be run up to an anode current $\left(I_{\mathrm{A}}\right)$ of $200 \mu \mathrm{A}$ but in practice their response in this region is not linear as a function of incident light intensity and we therefore limited our tube to a maximum current output of $20 \mu \mathrm{A}$ up to which we have verified its linearity. This limitation on $I_{\mathrm{A}}$ also ensures that $10 I_{\mathrm{A}}<I_{\mathrm{D}}$ the dynode chain current which further enhances the linearity of the response.

The photomultiplier was run with its cathode and mumetalshield (a precaution against stray electric and magnetic fields) at the negative HT whilst the anode was decoupled through the load resistor $R_{\mathrm{L}}$ to earth. The maximum negative HT used with this tube is $1500 \mathrm{~V}$ which was supplied by a Fluke model 415B HT supply which has an excellent stability of $0.002 \%$ per hour. The load resistor used depended on the dynamic range studied and this will be discussed below.

2) The anode output measured as a voltage across the load resistor $R_{\mathrm{L}}$,'was amplified and filtered before being sent to the transient recorder. This filter unit served both to amplify the signal by a factor of ten and to band limit the signal to frequencies below one half the sampling frequency which is the Nyquist frequency (i.e. $N_{q}=f_{\mathrm{s}} / 2$ ). This is a necessary precaution to avoid aliasing or the folding back of high frequency components above $N_{q}$ into the low frequency domain [13]. The filter which was home built was based on the Butterworth design [14]. Such a low pass filter has a maximally flat magnitude response which does not rise above its d.c. level. In our filter 5 stages of active filtering at a terminal slope of $12 \mathrm{~dB}$ per octave per stage were used to give a final terminal slope of $60 \mathrm{~dB} /$ octave. This gave an adequate attenuation factor and no aliasing was observed. Each filter train was preceded by an impedance matching stage for the load resistor $R_{\mathrm{L}}$, and frequency ranges (i.e. $N_{q}$ ) of $1,2,5,10,20$, and $50 \mathrm{kHz}$ were used. The frequency response of each filter train was linear and flat with $N_{q}$ being defined by the $-3 \mathrm{~dB}$ point. However when coupled to $R_{\mathrm{L}}$ and the Datalab recorder an apparatus function was observed. This function is obtained by measuring the white light spectrum of a d.c. heated incandescent filament. Such a source of random photons should give a flat spectral response. However as shown in figure 3 the response function changes regularly as a function of $R_{\mathrm{L}}$, and whilst using large values of $R_{\mathrm{L}}$ increases the gain of the system this involves a loss of frequency response which could induce large errors in the determination of the Power spectrum base line (see data analysis below). These errors could occur as the raw experimental power spectrum from an unknown polymer would have to be divided by a correction factor that is the normalized white light spectrum and at high frequencies this means dividing by a number that could be very small. We avoided such problems by limiting the gain and choosing a value of $R_{\mathrm{L}}$ that gave a flat response (within $\pm 2 \mathrm{~dB}$ ) up to the roll off at the Nyquist frequency.

3) After filtering the signal, which must be between $50 \mathrm{mV}$ and $50 \mathrm{~V}$, was amplified, digitized and stored in the Datalab DL901 transient recorder. The analog to digital conversion (ADC) with a resolution of 8 bits takes place in $5 \mu \mathrm{s}$. It is the sweep time of the transient recorder, between $5 \mathrm{~ms}$ and $200 \mathrm{~s}$ (in 1, 2, 5 steps) for the 1024 words, that fixes the frequency range selectable for the power spectra.

4) Under the command of a direct memory access parallel interface (DMA) the 1024 words are transferred from the recorder memory to the PDP 11/34 unit in $40 \mathrm{~ms}$. This minicomputer has $32 \mathrm{~K}$ words of store and a resolution of 16 bits. It is equipped with :

- a ten bit analog digital converter, two ten bit digital analog converters allowing communication with a graph plotter and an oscilloscope,

- a serial interface (current loop : $20 \mathrm{~mA}$ ) between the central unit and a teletype,

- two floppy disk units and one disk unit for the program and mass storage system.

The details of the interfaces that make the various components compatible in logic etc. are highly specialized and will be presented elsewhere.

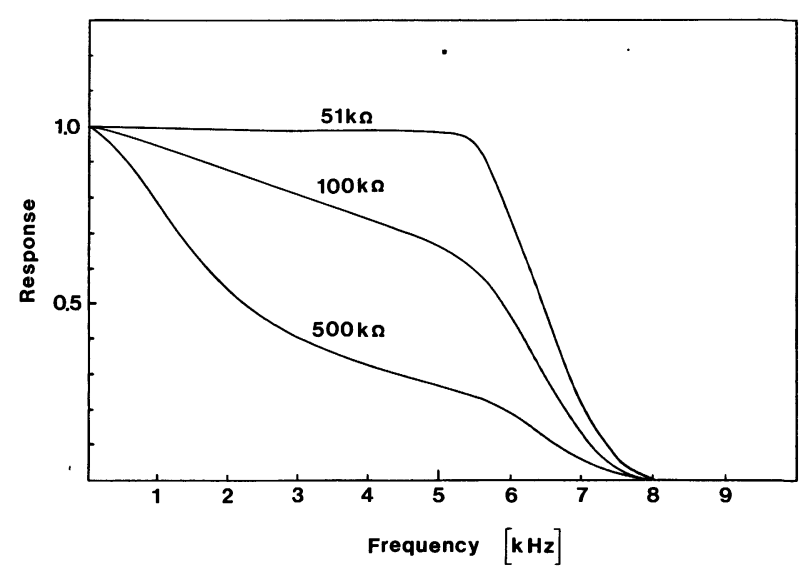

Fig. 3. - Apparatus response as a function of $R_{\mathrm{L}}$ for the $5 \mathrm{kHz}$ frequency range. The response functions were measured with white light using a Saicor 51B frequency analyser and are normalized at zero frequency. 
3.4 Data treatment. - As mentioned previously the Power Spectrum for a monodisperse scattering species will be a single Lorentzian of half width $I$ related to the diffusion constant $D_{t}$. In our apparatus this parameter is determined using a software approach directly from the time fluctuation of the scattered light intensity as recorded by the transient recorder. All operations are carried out by the PDP 11/34 using Assembler language and floating point simple precision under command from the teletype. The flow chart of this software approach is given in figure 4 and the key stages are as follows.

i) Start : All memories are cleared and the number of data points (up to 512) and the frequency range are specified. At this point a subroutine allows the programme to run up to the question saturation and permits the incoming data from the Datalab to be visualized via the PDP oscilloscope. Thus the amplification of the signal can be increased or decreased to ensure a full display within the 8 bit resolution (i.e. a full scale digital signal between 0 and 256) and allows the frequency range to be checked.

ii) Spectra Accumulation : after the selection of the number of accumulations $(S)$ to be made the

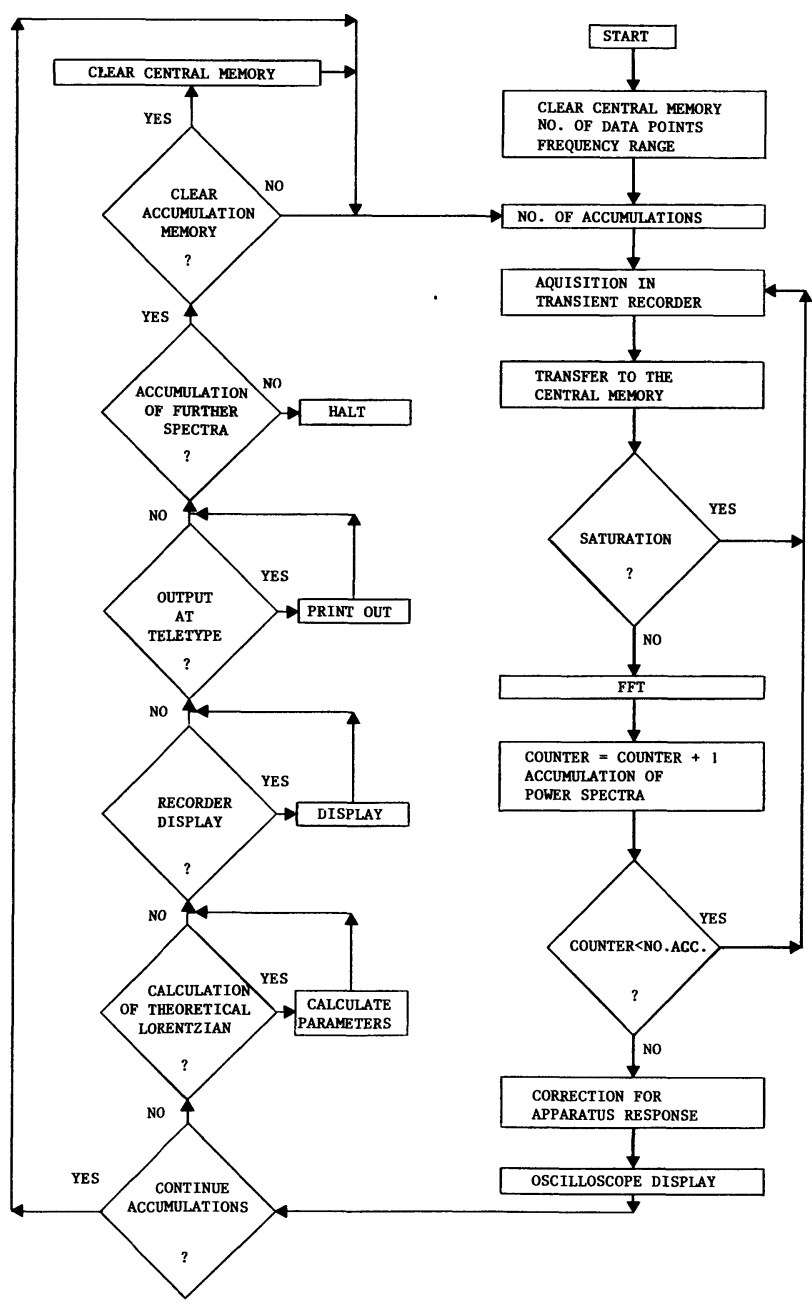

Fig. 4. - The software flow chart. programme steps through the cycle : - acquisition in the datalab memory, transfer to the control memory via DMA, examination for saturation in the signal which could occur if, for example dust passed through the scattering volume (in this case the signal would be rejected and the cycle returned to acquisition), FFT by the Tukey-Cooley algorithm, calculation of the power spectrum, and accumulation of these spectra in a separate memory until the preselected number of cycles has been made. Before this averaged spectrum is visually displayed it is corrected for the frequency response of the apparatus. This response is determined from the white light spectrum measured using an d.c. heated incandescent filament placed in the apparatus cell holder under the same apparatus conditions. Such a source of random photons should give a flat spectral response. However due to nonlinearities in the $R$ load line, filters and the Datalab this is not normally the case. Indeed each frequency range had a different apparatus function which was entered into an addressed buffer determined at the Start stage of the programme. The apparatus response corrections were carried out point by point using the smoothed white light spectrum determined after 10000 accumulations. After visual examination of the power spectrum further accumulations can be made or the curve fitting procedure selected.

iii) Curve fitting is carried out using a least mean square iterative procedure [15] supposing that the spectrum is single Lorentzian. Both the experimental and theoretical curves may then be printed out using the recorder display (see Fig. 6a). The constants of the theoretical Lorentzian and the point by point experimental values if required, are printed out at the teletype, along with an estimation of the standard error of the parameters and the root mean square deviation defined by

$$
\text { r.m.s. }=\left(\frac{1}{N-3} \sum_{i=1}^{N}\left[\frac{z_{i}-y_{i}}{y_{i}}\right]^{2}\right)^{1 / 2}
$$

where $z_{i}$ and $y_{i}$ are the experimental and theoretical value for the $i$ th spectral point. A test for alternance between the two curves is defined by :

$$
A=1-\frac{\sum_{i=1}^{N}\left(z_{i}-y_{i}\right)\left(z_{i+1}-y_{i+1}\right)}{\sum_{i=1}^{N}\left(z_{i}-y_{i}\right)^{2}} .
$$

The latter test allows a supplementary control of $\Gamma$ for normally accumulations are carried out until the r.m.s. value tends to a minimum and for a good fit $A$ tends to 1 whereas for a bad fit it tends to 0 . Normally values of $A>0.7$ combined with standard errors lower than $2 \%$ are acceptable [16]. At this point further spectra can be accumulated on top of those already in the memory or the complete cycle recommenced. 
Finally each $N$ point power spectrum cycle takes $0.8 \mathrm{~s}$ and the curve fitting and recording cycle takes roughly a minute.

3.5 SAMPLe PReparation. - The atactic polystyrene of low polydispersity $\left(M_{\mathrm{w}} / M_{\mathrm{n}} \sim 1.15\right)$ was prepared by anionic polymerization and had weight average molecular weights of $1.39 \times 10^{6}$ and $1.4 \times 10^{5}$ daltons and solutions were made using triply distilled benzene. The polystyrene latex spheres of nominal diameter $91 \mathrm{~nm}$ were obtained from Dow Chemicals Ltd and suspension were made at neutral $\mathrm{pH}$ in doubly distilled deionized water. All solutions or suspensions were filtered several times using millipore filters of $0.45 \mu \mathrm{m}$ pore size. The criterion for a dust free sample was that the integrated scattering signal at $20^{\circ}$ was stable to within $1 \%$ after successive filtering.

4. Results. - 4.1 Static alignment and CaliBRATION. - Before $R_{\theta}$ can be determined from the angular scattering profile it is necessary to verify the symmetry of the apparatus and the correction for the scattering volume as a function of angle. This is normally done using benzene or a fluorescent solution [1], and for vertically polarized incident light [8] the volume correction should be simply $\sin \theta$ and the symmetry is then given by $I_{\theta} \sin \theta / I_{\theta=90^{\circ}}$. From table $I$ it can be seen that the ratio is unity within a few percent for all angles between $20^{\circ}$ and $140^{\circ}$ and thus the apparatus is correctly aligned and the volume correction appropriate.

In order to verify the calibration of the apparatus, using benzene as the calibrating reference, static light scattering measurements were carried out on a polystyrene of known molecular weight

$$
\left(M_{\mathrm{w}}=1.39 \times 10^{6}\right)
$$

in benzene, and using the Zimm plot method, figure 5, the constants listed in table II were obtained. All lines of constant concentration $c$ and angle $\theta$ were calculated from the data points and extrapolated to zero $c$ and $\theta$ using the least squares fitting procedure of the main programme, and from the least squares fitted lines of zero $c$ and $\theta$ the values of $M_{\mathrm{w}},\left(R_{\mathrm{g}}^{2}\right)^{1 / 2}$ and $A_{2}$ were calculated. In order to compare the performance of the current apparatus with other commercial instruments we have reproduced in table II the molecular constants recently measured [8] for the
Table II. - Comparison of the molecular parameters determined for a polystyrene sample in benzene at $25^{\circ} \mathrm{C}$, between the current apparatus and two commercial instruments. $\lambda_{0}$ is the incident scattering wavelength

\begin{tabular}{|c|c|c|c|c|}
\hline Instrument & $\begin{array}{c}\lambda_{0} \\
(\mathrm{~nm})\end{array}$ & $\begin{array}{c}M_{w} \cdot 10^{-6} \\
\text { daltons }\end{array}$ & $\begin{array}{c}\left\langle R_{\mathrm{g}}^{2}\right\rangle^{1 / 2} \\
(\mathrm{~nm})\end{array}$ & $\frac{A_{2}}{\mathrm{~mol} \cdot \mathrm{m}^{3} \cdot \mathrm{kg}^{-2}}$ \\
\hline - & - & - & - & - \\
\hline Current & 514.6 & $1.39_{8}$ & 71.1 & 3.99 \\
\hline FICA 50 & 546 & $1.40_{5}$ & 68.0 & 3.6 \\
\hline FICA 42000 & 632 & 1.42 & 70.0 & 3.4 \\
\hline
\end{tabular}

same polymer in benzene on two different SOFICA instruments. From the accord between the constants we conclude that in using collecting optics that see only a small part of the scattering volume then the correction that could arise from the complex shape of the beam waist at the focal point is negligible and that the apparatus may be used for static as well as dynamic measurements.

4.2 Dynamic Tests and Performance. - In order to test the correct functioning of the FFT system measurements have been carried out on calibrated polystyrene latex spheres at a suspension concentration of $10^{-2} \mathrm{~g} \mathrm{.} \mathrm{cm}^{-3}$ for scattering angles between $20^{\circ}$ and $130^{\circ}$ and temperatures between $15^{\circ} \mathrm{C}$ and $55^{\circ} \mathrm{C}$. The half width $(\Gamma)$ for each spectrum determined from the least squares fit of the single Lorentzian, see figure $6 a$, is given for 1000 scan runs as a function of $q^{2} / \pi$ in figure $6 b$. From the slope of each of these plots the diffusion constant $D_{t}$ was determined (using the least squares procedure) as a function of temperature and these constants (which were independent of concentration) and the values of the particle diameter $(d)$ determined from them using the general Stokes-Einstein equation are given in table III. Following this equation i.e.

$$
d=k_{\mathrm{b}} T\left(3 \pi \eta D_{\mathrm{t}}(0)\right)^{-1},
$$

where $k_{\mathrm{b}}$ is the Boltzmann constant, $T$ the absolute temperature, $\eta$ the solvent viscosity and $D_{t}(0)$ the diffusion constant extrapolated to zero concentration, the values of $\eta D_{t}(0)$ have been curve fitted as a linear function of $T$. From the slope of this line the mean value of $d$ listed in table III has been calculated. This compares well with a value of $d=91.0 \mathrm{~nm}$

Table I. - Verification of the scattering volume as a function of $\sin \theta$ and the symmetry of the apparatus using

\begin{tabular}{|c|c|c|c|c|c|c|c|c|c|c|c|c|c|}
\hline$\theta$ & $20^{\circ}$ & $30^{\circ}$ & $40^{\circ}$ & $50^{\circ}$ & $60^{\circ}$ & $70^{\circ}$ & $80^{\circ}$ & $90^{\circ}$ & $100^{\circ}$ & $11^{\circ}$ & $120^{\circ}$ & $130^{\circ}$ & $140^{\circ}$ \\
\hline- & - & - & - & - & - & - & - & - & - & - & - & - & - \\
\hline$\frac{I \sin \theta}{I_{\theta=90^{\circ}}}$ & $0.97_{1}$ & $0.99_{9}$ & $0.99_{8}$ & $1.00_{5}$ & $1.00_{3}$ & $1.01_{5}$ & $1.01_{6}$ & 1 & $0.97_{7}$ & $1.00_{6}$ & $0.99_{2}$ & $1.01_{8}$ & \\
\hline
\end{tabular}
benzene as the scattering medium. 


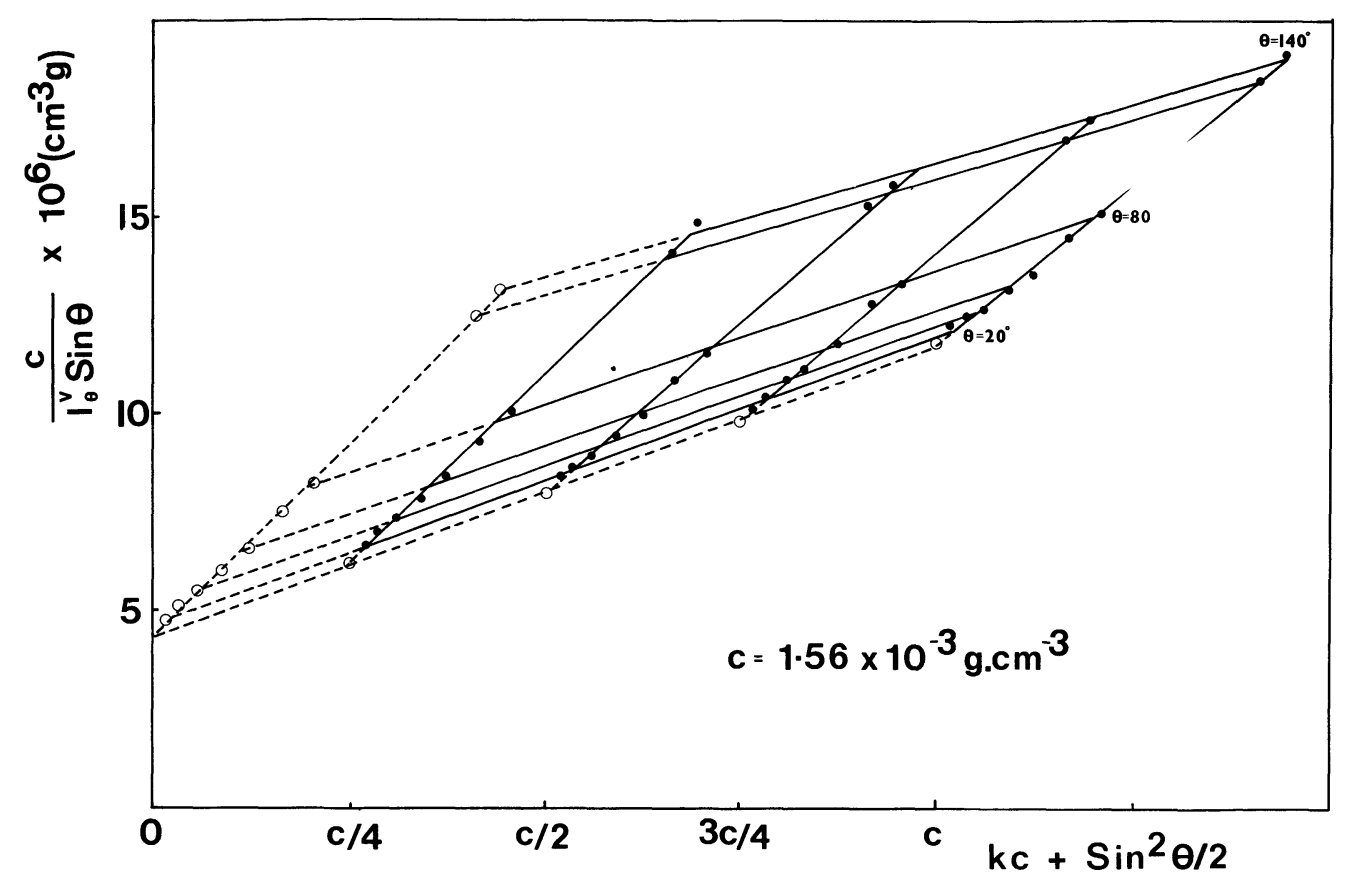

Fig. 5. - Zimm plot for polystyrene $\left(M_{\mathrm{w}}=1.4 \times 10^{6}\right)$ in benzene at $25^{\circ} \mathrm{C}$. The full points and lines are experimental and the hollow circles and dashed lines are extrapolated. All lines of constant $c$ and $\theta$ have been fitted by the least mean squares procedure. For clarity not all of the * lines of constant $\theta$ have been given, and values of $I_{\mathrm{b}}^{v}=20, \frac{\mathrm{d} n}{\mathrm{~d} c}=0.107 \mathrm{~cm}^{3} \cdot \mathrm{g}^{-1}$, and $n_{0}=1.51$ have been used in the calculations.

a)
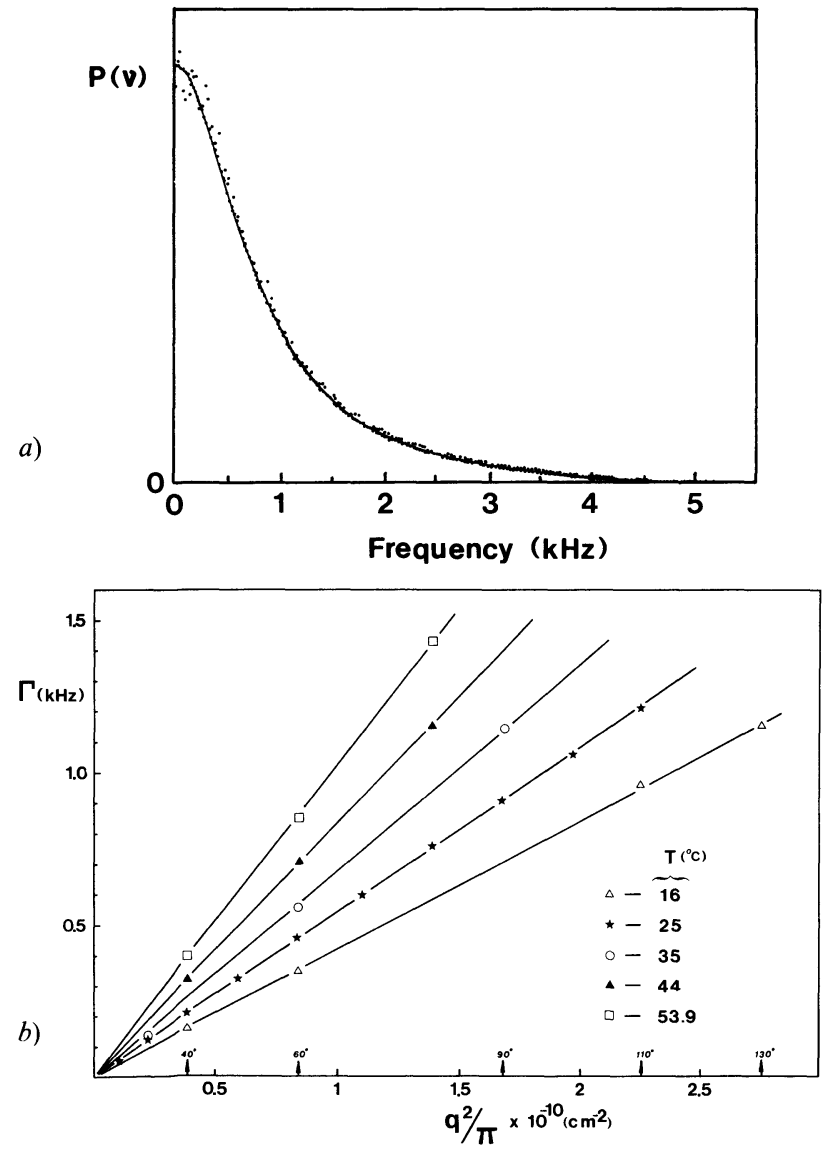

Fig. 6. - $-a$ ) Typical power spectrum in arbitrary units of the light scattered from the polystyrene latex spheres for an angle of observation of $90^{\circ}$ at $T=23{ }^{\circ} \mathrm{C}$ and $\lambda_{0}=514.5 \mathrm{~nm}$. The points are experimental and the line is the least mean squares fitted lorentzian $(\Gamma=850 \mathrm{~Hz}) . b)$ Spectral half widths $\Gamma$ as a function of $q^{2} / \pi$ for the polystyrene latex spheres at various temperatures.
Table III. - Mean dynamic constants of the polystyrene/water suspension. We estimate uncertainties of $\pm 2 \%$ to be associated with the values of $D_{\mathrm{t}}$

$\begin{array}{lcccccc}T\left({ }^{\circ} \mathrm{C}\right) & 16 & 25 & 35 & 44 & 53.9 & \text { Mean } \\ D_{\mathrm{t}} \times 10^{-8} \mathrm{~cm}^{2} / \mathrm{s} & 4.19 & 5.41 & 6.79 & 8.34 & 10.28 & - \\ d(\mathrm{~nm}) & 91.2 & 90.6 & 92.4 & 91.6 & 90.8 & 91.2\end{array}$

(with a standard deviation of $5.8 \mathrm{~nm}$ ) given by the supplier.

Given the consistency of the two independent sets of results we conclude that the dynamic apparatus is well calibrated both in angle and as a function of temperature over the frequency range studied. For such polystyrene latex particles the frequency spectra are limited to the low frequency ranges of the apparatus (i.e. up to $10 \mathrm{kHz}$ ) and in order to test the higher frequency response it is necessary to find a monodisperse scattering species that diffuses more rapidly. Unfortunately stable polystyrene latex particles much smaller than those used are not commercially available. However the problem can be resolved by using low molecular weight polystyrene in a random coil promoting solvent provided the sample is suitably monodisperse. Using a polystyrene of $M_{\mathrm{w}}=140000$ in benzene at a concentration of $8.8 \times 10^{-3} \mathrm{~g} / \mathrm{cc}$ the power spectra half widths as a function of $q^{2} / \pi$ shown in figure 7 were recorded. From the good linearity of this plot we conclude that the frequency ranges up to $50 \mathrm{kHz}$ are responding correctly. From the slope of this line a value of $D_{\mathrm{t}}=1.33 \times 10^{-7} \mathrm{~cm}^{2} \mathrm{~s}^{-1}$ was determined. As will 


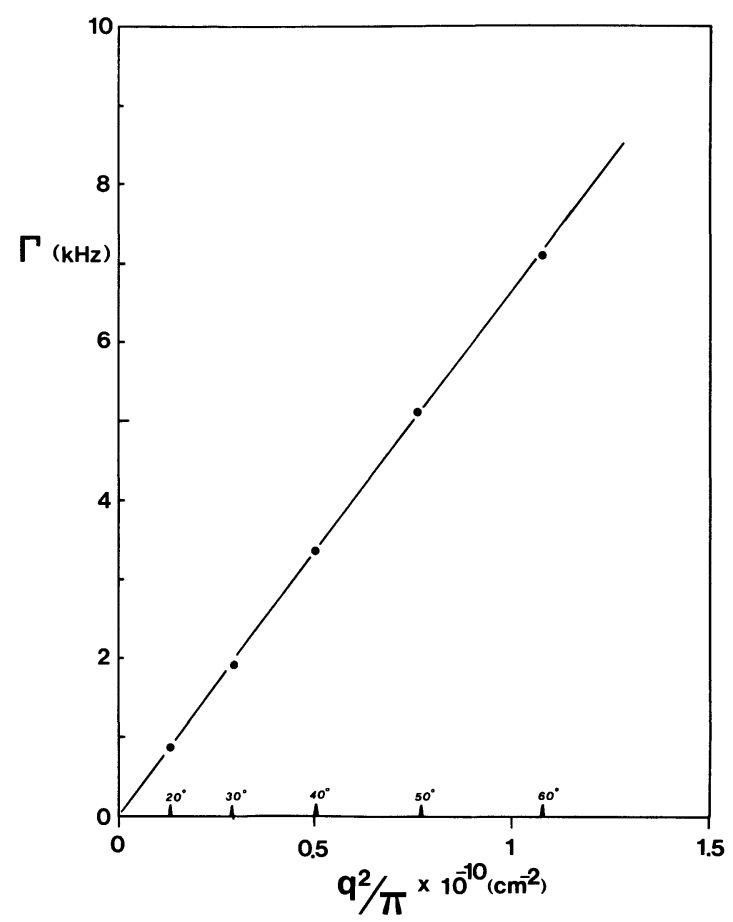

Fig. 7. - Spectral half width $\Gamma$ as a function of $q^{2} / \pi$ for the polystyrene of $M_{\mathrm{w}}=140000$ in benzene at $25^{\circ} \mathrm{C}$ for

$$
c=8.8 \times 10^{-3} \mathrm{~g} / \mathrm{cm}^{3} \text {. }
$$

be discussed below for interacting polymer solutions $D_{\mathrm{t}}$ is a function of concentration and as this is the case for polystyrene in benzene the value of $D_{\mathrm{t}}$ quoted is specific to the concentration used.

4.3 A FLEXIBLE POLYMER SYSTEM. - The translational diffusion constant for dilute but interacting polymer solutions can be expanded to the first order in concentration as [17]

$$
D_{\mathrm{t}}(c)=D_{\mathrm{t}}(0)\left(1+K_{\mathrm{D}} c+\cdots\right)
$$

where $D_{\mathrm{t}}(0)\left(=k_{\mathrm{b}} T / 6 \pi \eta R_{\mathrm{H}}\right)$ is the diffusion constant at infinite dilution and the other terms are as previously defined. In this model the polymers are considered as spheres of hydrodynamic radius $R_{\mathrm{H}}$ and the term $K_{\mathrm{D}}$ takes into account the repulsive (i.e. excluded volume) and hydrodynamic interactions. Following this model with the molecules treated as hard spheres, $K_{\mathrm{D}}$ may be related to the same effective radius $R_{\mathrm{H}}$ (provided $c$ is in units of number concentration i.e. $\mathrm{cm}^{-3}$ ) by : $K_{\mathrm{D}}=8 \pi R_{\mathrm{H}}^{3} / M$ where $M$ is the molecular weight. Further following Flory [18] the second virial coefficient $A_{2}$ is given by $A_{2}=16 \pi N_{\mathrm{A}} R_{\mathrm{e}}^{3} / 3 M^{2}$ where $R_{\mathrm{e}}$ is the radius of an equivalent hard sphere that is less than the radius of gyration (see sects. 12 and 20 of reference [2]).

Using the PS sample of $M_{\mathrm{w}}=1.4 \times 10^{6}$ daltons previously characterized by the static experiment we have measured the spectral half widths $\Gamma$ as a function of $q^{2} / \pi$ and concentration, figure 8. From the slope of these linear functions $D_{t}(c)$ has been calculated and is given as a function of concentration in figure 9 . Clearly $D_{t}(c)$ is a linear function in $c$ for this polymer thus confirming the theoretical predictions [17]. Using a least squares fit the values of $D_{\mathrm{t}}(0)$ and $K_{\mathrm{D}}$ were determined and the values of $R$ calculated from them along with that determined from $A_{2}$ are given in table IV. Within the experimental uncertainty these values appear to be approximately the same, although following reference [20] the value

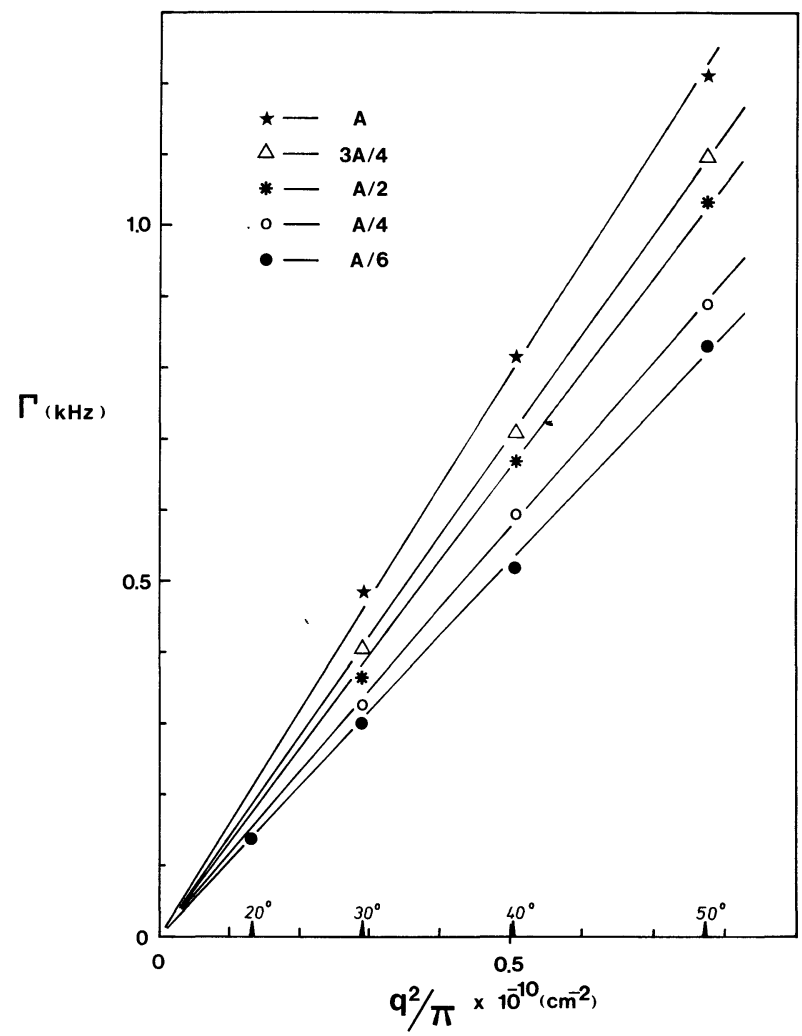

Fig. 8. - Spectral half widths $\Gamma$ as a function of $q^{2} / \pi$ for polystyrene of $M_{\mathrm{w}}=1.4 \times 10^{6}$ in benzene for various concentrations $A=3.646 \times 10^{-3} \mathrm{~g} / \mathrm{g}$.

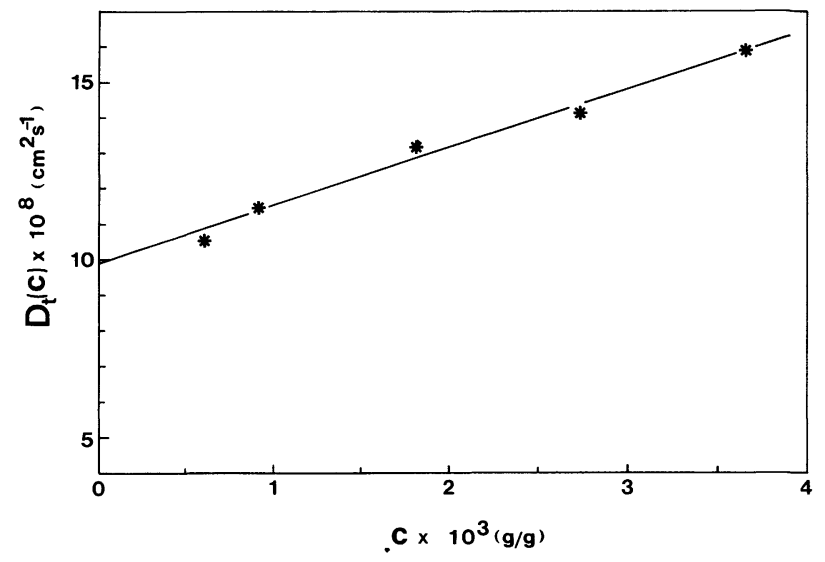

Fig. 9. - Diffusion constants $D_{\mathrm{t}}(c)$ as a function of $c$ for polystyrene $\left(M_{\mathrm{w}}=1.4 \times 10^{6}\right)$ in benzene solutions. 
Table IV. - Theoretical hard sphere radius $(R)$ for polystyrene of $M_{\mathrm{w}}=1.4 \times 10^{6}$ in benzene at $25^{\circ} \mathrm{C}$. The uncertainties associated with values of $R$ are estimated to be $\pm 5 \%$.

$\begin{array}{cccc}\text { Experiment } & D_{\mathrm{t}}(0) & K_{\mathrm{D}} & A_{2} \\ (R) \mathrm{nm} & 36.5 & 36.0 & 38.9\end{array}$

of $R$ determined from $K_{\mathrm{D}}$ may be equivalent to some average involving both $\left(R_{\mathrm{g}}^{2}\right)^{1 / 2}$ and $R_{\mathrm{H}}$. Further discussion of this point is outside the scope of the current article although it does suggest that a careful study of both $R_{\mathrm{H}}$ and $\left(R_{\mathrm{g}}^{2}\right)^{1 / 2}$ as determined from the dynamic and static techniques should be made on identical samples as a function of Molecular Weight. From table IV it is clear that the value of $R_{\mathrm{H}}$ determined from $D_{t}(0)$ is approximately half that determined for the static radius of gyrations $\left(R_{\mathrm{g}}^{2}\right)^{1 / 2}$ (see table II). As was recently discussed in detail [19] static and dynamic experiments give polymer dimensions that appear to differ. Indeed in good solvents far from the $\theta$ conditions it has already been shown that [20] $R_{\mathrm{H}} \propto M^{0.55}$ and [21] $\left(R_{\mathrm{g}}^{2}\right)^{1 / 2} \propto M^{0.6}$. From our preliminary results we conclude that at high enough molecular weight these differences are readily observed. Finally from an experimental viewpoint, the values of $D_{t}(0)$ and $K_{\mathrm{D}}$ are in good agreement with those determined over a wide molecular weight range using a hardware Malvern correlator system [20].

5. Conclusion. - A self contained light scattering apparatus capable of performing static (i.e. total intensity) and dynamic measurements has been described and tested. Measurements have been carried out on calibrated polystyrene latex spheres and flexibly polystyrene in benzene solutions and these results compared with theoretical predictions. The thermal stability and angular scattering calibration were rigorously tested and good agreement found between the static and dynamic measurements. The performance of the current apparatus compares favourably with commercially available static and dynamic light scattering apparatuses.

Acknowledgments. - The authors would like to thank most sincerely Dr R. Duplessix for his initial enthusiasm in setting up the project, Dr C. Picot for numerous discussions, and F. Woehl and R. Bersinger for their inestimable support in fabrication of the electronic and mechanical components respectively.

\section{References}

[1] Huglin, M. B. (Ed.), Light Scattering from Polymer Solutions (Academic Press, London and New York) 1972.

[2] TANFord, C., Physical Chemistry of Macromolecules (Wiley, New York) 1961.

[3] Berne, B. and Pecora, R., Dynamic Light Scattering (Wiley, New York) 1976.

[4] Chu, B., Laser Light Scattering (Academic Press, London and New York) 1974.

[5] Jolly, D. and Eisenberg, H., Biopolymers 15 (1976) 61.

[6] Cummins, H. Z. and Pike, E. R. (Eds), Photon Correlation and Light Beating Spectroscopy (Plenum Press) 1974.

[7] Utiyama, H., Chapter 3 of reference [1].

[8] Millaud, B. and Strazielle, C., Macromol. Chem. 180 (1979) 441.

[9] Cooley, J. W. and Tukey, J. W., Math. Comput. 19 (1965) 297.

[10] Shaya, S. A., Han, C. C. and Yu, H., Rev. Sci. Instrum. 45 (1974) 280.

[11] Chu, B. and Schoenes, F. J., J. Colloid Sci. 27 (1968) 424.
[12] Jamieson, A. M. and Walton, A. G., J. Chem. Phys. 58 (1973) 1054.

[13] Randall, R. B., in Frequency Analysis (Brüel \& Kjaer Publications) 1977.

[14] Martin, A. G. and Stephenson, F. W., Chap. 8 in Linear Microelectronic Systems (Mac-Millan Press, London) 1973.

[15] Kim, H., J. Chem. Educ. 47 (1970) 120.

[16] Delsanti, M. L. H., Thesis, Université de Paris-Sud, 1978.

[17] Altenberger, A. R. and Deutch, J. M., J. Chem. Phys. 59 (1973) 894.

[18] Flory, P. J., Principles of Polymer Chemistry (Cornell Univ. Press) 1953.

[19] Weill, G. and des Cloizeaux, J., J. Physique 40 (1979) 99.

[20] Adam, M. and Delsanti, M. L. H., J. Physique 37 (1976) 1045.

[21] Cotton, J. P., Decker, D., Farnoux, B., Jannink, G., Ober, R. and Picot, C., Phys. Rev. Lett. 32 (1974) 1170. 